\title{
A Design Method for Fault Reconfiguration and Fault-Tolerant Control of a Servo Motor
}

\author{
Jing He $\mathrm{He}^{1,2}$ and Changfan Zhang ${ }^{1}$ \\ ${ }^{1}$ College of Electrical and Information Engineering, Hunan University of Technology, Zhuzhou, Hunan 412007, China \\ ${ }^{2}$ College of Mechatronic Engineering and Automation, National University of Defense Technology, Changsha, Hunan 410073, China
}

Correspondence should be addressed to Changfan Zhang; zhangchangfan@263.net

Received 13 February 2013; Accepted 8 July 2013

Academic Editor: Yuqiang Wu

Copyright (c) 2013 J. He and C. Zhang. This is an open access article distributed under the Creative Commons Attribution License, which permits unrestricted use, distribution, and reproduction in any medium, provided the original work is properly cited.

A design scheme that integrates fault reconfiguration and fault-tolerant position control is proposed for a nonlinear servo system with friction. Analysis of the non-linear friction torque and fault in the system is used to guide design of a sliding mode position controller. A sliding mode observer is designed to achieve fault reconfiguration based on the equivalence principle. Thus, active fault-tolerant position control of the system can be realized. A real-time simulation experiment is performed on a hardware-in-loop simulation platform. The results show that the system reconfigures well for both incipient and abrupt faults. Under the fault-tolerant control mechanism, the output signal for the system position can rapidly track given values without being influenced by faults.

\section{Introduction}

Modern wars require rapid troop mobility over a large combat field. This means that ground operation platforms have to accurately determine the current position and must also possess ground navigation ability [1]. Electromechanical tracking and stable servo platforms are designed based on this requirement. The platform consists of posture sensors, a main control unit, a servo control unit, a precision servo mechanism, and a signal receiving unit. The antenna is jointly controlled by rotation on a horizontal axial system (azimuth control) and on a vertical axial system (pitch control). Both axial systems use independent servo control systems, a transmission system, and a rotary actuator.

In the servo control unit, both the azimuth and pitch motors are DC servo motors. The inverting problem is complicated from a theoretical viewpoint and is constrained by operating conditions in actual applications. Multiple faults such as electrical brush faults, open circuits in component, interturn short circuits, and coil resistance caused by temperature increases may occur during operation, which leads to difficulties in system maintenance of DC motors. Friction is almost ubiquitous in servo systems. Nonlinear friction torque impacts greatly the dynamic and static performance of a system. As a consequence, the output response will have a large static error or produce steady-state limit-cycle oscillations. The influence on dynamic performance is manifest as the creep phenomenon at low speed $[2,3]$. When static friction is greater than Coulomb friction, a conventional PI controller cannot eliminate the static error. Instead, it will cause a limit oscillation of the system response $[4,5]$. Hence, high-precision position servo systems require a better controller to cancel out the influence of friction torque to yield higher system precision, a quicker response, and lower overshoots and static errors [6, 7]. It is well known that sliding mode control (SMC) is attracting a significant interest in servo system application research duo to its simplicity in application and good performance. Utkin's book [8] is fully dedicated to SMC in this subject. Šabanovic [9] presents a comprehensive overview of the application of SMC methods in motion control systems.

In this paper, a servo control unit is investigated for electromechanical tracking and a steady servo platform. The effect of faults and nonlinear friction torque in the DC servo motor control system is first analyzed. Then an SMC is used for control of the DC azimuth and pitch servo motors, taking advantage of its nonlinear processing capacity. On this basis, a fault reconfiguration algorithm and an algorithm 
for rectifying the control parameters using the output of an observer are developed, both of which contribute to achieving active fault-tolerant control (FTC) of the system.

The remainder of the paper is organized as follows: Section 2 describes the servo motor system. Section 3 proposes the scheme for fault reconstruction and FTC. Experimental results are presented in Section 4, with conclusions in Section 5.

\section{System Description}

Under normal conditions, DC servo motors used for azimuth and pitch control can be simplified into linear second-order elements. However, under low-speed conditions, the system shows severe nonlinearity because of strong friction. The system can be described by [10]

$$
\begin{gathered}
\dot{x}_{1}=x_{2}, \\
\dot{x}_{2}=-\frac{K_{m} C_{e}}{J R} x_{2}+K_{u} \frac{K_{m}}{J R} u-\frac{1}{J} F_{f}\left(x_{2}, t\right), \\
y=x_{1},
\end{gathered}
$$

where $x_{1}$ and $x_{2}$ represent the electrical angular position and speed, respectively, and $K_{u}$ is the amplification factor of the power amplifier, $R$ is the armature resistance, $K_{m}$ is the motor moment coefficient, $C_{e}$ is the voltage feedback coefficient, $J$ is the moment of inertia, $u$ is the control input, and $y$ is the output. $F_{f}\left(x_{2}, t\right)$ is the friction, given by the following [11].

When $\left|x_{2}\right|<a$, the static friction is

$$
F_{f}\left(x_{2}, t\right)= \begin{cases}F_{m} & F(t)>F_{m}, \\ F(t) & -F_{m}<F(t)<F_{m}, \\ -F_{m} & F(t)<-F_{m} .\end{cases}
$$

When $\left|x_{2}\right|>a$, the dynamic friction is

$$
\begin{gathered}
F_{f}\left(x_{2}, t\right)=\left[F_{c}+\left(F_{m}-F_{c}\right) e^{-a_{1}\left|x_{2}\right|}\right] \operatorname{sgn}\left(x_{2}\right)+k_{v} x_{2} \\
F(t)=J \dot{x}_{2},
\end{gathered}
$$

where $F(t)$ is the driving force, $F_{m}$ is the maximum static friction, $F_{c}$ is Coulomb friction, $k_{v}$ is the proportionality factor for viscous friction torque, and $a$ and $a_{1}$ are very small positive constants.

Assume that $k_{1}=K_{m} C_{e} / J R, k_{2}=K_{u}\left(K_{m} / J R\right)$, and $k_{3}=1 / J$ are three nominal values. When considering the occurrence of faults in the controlled object, the following equation is obtained:

$$
F_{a}=-\Delta k_{1} x_{2}+\Delta k_{2} u
$$

Then (1) can be written as

$$
\begin{gathered}
\dot{x}_{1}=x_{2}, \\
\dot{x}_{2}=-k_{1} x_{2}+k_{2} u-k_{3} F_{f}\left(x_{2}, t\right)+F_{a}, \\
y=x_{1},
\end{gathered}
$$

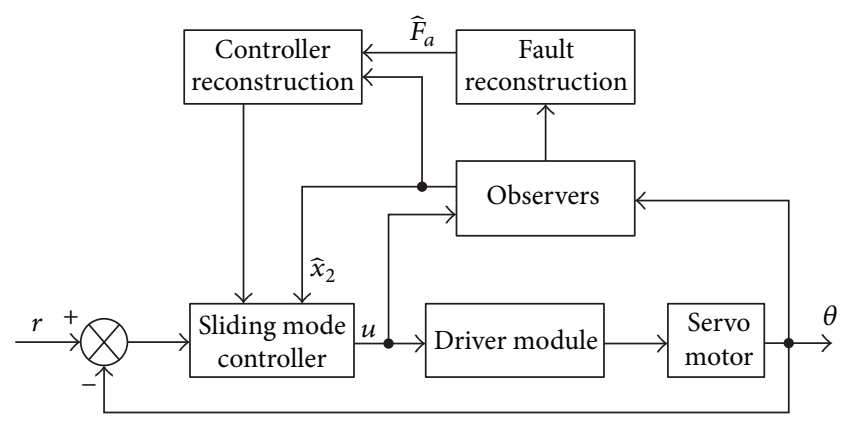

FIGURE 1: Structural diagram of FTC of a servo motor.

where $\Delta k_{1}$ and $\Delta k_{2}$ are faults leading to variations in parameters such as $K_{m}$ and $R$. Normally these are zero; otherwise, they are unknown nonlinear functions that can be described by the generalized fault in (4).

Actuator faults involve sticking, constant-gain variations or constant errors, and time-varying errors for execution of order in the control circuit. Then the input signal becomes $u(t)+f_{a}(t)$, where $f_{a}(t)$ is the actuator fault. Equation (5) can still be used to describe a system with an actuator fault, and then the generalized fault would be $F_{a}=k_{2} f_{a}(t)$.

\section{FTC Algorithm for a Servo Motor}

To ensure that the azimuth and pitch axis stabilization and tracking platform can operate reliably during combat missions, the tracking precision of the antenna should be guaranteed under all circumstances. Therefore, the most important purpose of fault diagnosis is to ensure that the system can maintain accurate tracking of the azimuth and pitch motors for a given angle, even under a fault condition, that is, to implement FTC for the system.

FTC is divided into passive and active fault tolerance for the control mechanism. Passive FTC only takes controller robustness into consideration and can assure system performance to a certain extent. However, its self-adaptive ability related to fault timing and amplitude is poor, so passive FTC has a limited fault tolerance capacity. On the contrary, active FTC readjusts the controller parameters after a fault has taken place and can even change the structure of a controller if necessary. Thus, it can greatly increase the system performance [12]. In this paper, fault reconfiguration is first constructed on the basis of a variable structure for the sliding mode, which leads to simultaneous active FTC for the servo motor. The general structure of the system is shown in Figure 1.

In Figure 1, $r$ is given by the system, $u$ is the input of the controlled object, and $\theta$ is the electrical angular position, detected by an angular displacement sensor. $\widehat{F}_{a}$ is the estimation (or reconfiguration) of the fault provided by the fault reconfiguration mechanism, and $\widehat{x}_{2}$ is the estimation of $x_{2}$.

For the driving module and nonlinear system for the servo motor shown in Figure 1, a measurable signal $u$ and output $\theta$ are used to construct a state observer. On one 
hand, fault reconfiguration is realized; on the other hand, the estimation of the fault and other state variables are used to rectify the parameters of the SMC to achieve active FTC. Besides, the system utilizes only one sensor, which is the angular displacement sensor in the servo motor. This method reduces the number of sensors and has strong practicality for industrial application.

3.1. Design of the Sliding Mode Controller. The advantage of SMC is that when a sliding mode occurs on a surface, the system has better invariance and robustness with regard to uncertainties such as modeling errors, parameter variations, and disturbances [13]. Therefore, SMC has greatly improved the robustness of fault diagnosis systems [14]. In addition, the SMC algorithm is simple and easy to implement for real-time engineering. Therefore, the SMC is first designed.

We define the control error as $e=r-x_{1}$ and choose the sliding surface as

$$
s=c e+\dot{e}=c\left(r-x_{1}\right)+\dot{r}-x_{2},
$$

where $c>0$.

When no fault has occurred (i.e., $F_{a}=0$ ), the reaching law is used as the basis for SMC [14]. The SMC is

$$
u=\frac{J R}{K_{u} K_{m}}\left(c \dot{e}+\dot{r}+\varepsilon \operatorname{sgn}(s)+k s+\frac{K_{m} C_{e}}{J R} x_{2}+\frac{F_{f}\left(x_{2}, t\right)}{j}\right),
$$

where $k>0$.

It is evident from (7) that the system state variable is one of the basic prerequisites for accomplishing control. Thus, the key to realizing control is design of an effective state observer. When a fault occurs, FTC has to be accomplished in combination with fault evaluation.

3.2. FTC Design. Considering that the state variable $x_{2}$ in (7) is immeasurable and that a fault influences the system, the following state observer and FTC are proposed using input and output signals.

First, the sliding mode observer for the system in (5) is

$$
\begin{gathered}
\dot{\hat{x}}_{1}=\widehat{x}_{2}+L_{1} \operatorname{sgn}\left(x_{1}-\widehat{x}_{1}\right), \\
\dot{\hat{x}}_{2}=-k_{1} \widehat{x}_{2}+k_{2} u-k_{3} F_{f}\left(\widehat{x}_{2}, t\right)+L_{2} \operatorname{sgn}\left(x_{2}-\widehat{x}_{2}\right),
\end{gathered}
$$

where $\wedge$ denotes the observed value of the corresponding variable and $L_{1}$ and $L_{2}$ are the coefficients to be designed $\left(L_{1}, L_{2}>0\right)$.

If the errors of the observer and the fault reconfiguration are defined as $e_{1}=x_{1}-\widehat{x}_{1}$ and $e_{2}=x_{2}-\widehat{x}_{2}, \bar{F}_{f}=F_{f}\left(x_{2}, t\right)-$ $F_{f}\left(\widehat{x}_{2}, t\right)$, then the state estimation error dynamics can be obtained from (5) and (8):

$$
\begin{gathered}
\dot{e}_{1}=\dot{x}_{1}-\dot{\hat{x}}_{1}=e_{2}-L_{1} \operatorname{sgn}\left(x_{1}-\widehat{x}_{1}\right), \\
\dot{e}_{2}=\dot{x}_{2}-\dot{\hat{x}}_{2}=-k_{1} e_{2}-k_{3} \bar{F}_{f}+F_{a}-L_{2} \operatorname{sgn}\left(x_{2}-\widehat{x}_{2}\right) .
\end{gathered}
$$

$V_{1}=(1 / 2) e_{1}^{2}$ is chosen as the Lyapunov function. Along the trajectory of system (9), the derivative of the Lyapunov function with respect to time is

$$
\dot{V}_{1}=e_{1} \dot{e}_{1}=e_{1}\left(e_{2}-L_{1} \operatorname{sgn} e_{1}\right) \leqslant\left|e_{1}\right|\left(\left|e_{2}\right|-L_{1}\right) .
$$

If $L_{1}$ is chosen such that $L_{1}>\left|e_{2}\right|+\eta_{1}$ and $\eta_{1}>0$, then $\dot{V}_{1} \leq-\eta_{1}\left|e_{1}\right|$. Then, after a limited time $t_{1}$, the sliding mode is reached. Then $e_{1}=\dot{e}_{1}=0$ can be obtained based on the equivalent principle for the sliding mode variable structure [15]. That is,

$$
\begin{gathered}
\forall t>t_{1}, \quad \widehat{x}_{1}=x_{1}, \\
\left(L_{1} \operatorname{sgn} e_{1}\right)_{\mathrm{eq}}=e_{2},
\end{gathered}
$$

where $\left(L_{1} \operatorname{sgn} e_{1}\right)_{\mathrm{eq}}$ is the equivalent output injection value of the discontinuous term, which is used to estimate $e_{2}$.

Next, $V_{2}=(1 / 2)\left(e_{1}^{2}+e_{2}^{2}\right)$ is chosen as the Lyapunov function.

By considering the previous steps, the time derivative of $V_{2}$ along the trajectories of system (10) is given by

$$
\begin{aligned}
\dot{V}_{2} & \leq e_{2} \dot{e}_{2}=e_{2}\left(-k_{1} e_{2}-k_{3} \bar{F}_{f}+F_{a}-L_{2} \operatorname{sgn}\left(e_{2}\right)\right) \\
& \leq-k_{1} e_{2}^{2}+\left|e_{2}\right|\left(\left|F_{a}-k_{3} \bar{F}_{f}\right|-L_{2}\right) .
\end{aligned}
$$

Assuming that $F_{a}$ is a bounded function and the system has input-to-state stability before and after the fault; then $\bar{F}_{f}$ is also a bounded function. Hence, a large enough $L_{2}$ is chosen to make (14) negative definite; that is, $L_{2}>\left|F_{a}\right|+$ $k_{3}\left|\bar{F}_{f}\right|$, and $e_{2} \dot{e}_{2} \leq-k_{1} e_{2}^{2}$ is obtained.

Similarly, according to the equivalent principle for the sliding mode variable structure, it follows that a sliding motion takes place in finite time and during the sliding motion

$$
\forall t>t_{2}, \quad e_{2}=\dot{e}_{2}=0, \quad t_{2}>t_{1}
$$

and thus from (10)

$$
F_{a}=L_{2} \operatorname{sgn}\left(e_{2}\right) .
$$

To reduce chattering in the sliding mode variable structure and eliminate high-frequency interference, a continuous function can be used to replace the sign function in (13) and (16) [16]. The fault reconfiguration can then be expressed as

$$
\begin{aligned}
& \widehat{e}_{2}=L_{1} \frac{e_{1}}{\left|e_{1}\right|+\delta_{1}}, \\
& \widehat{F}_{a}=L_{2} \frac{\widehat{e}_{2}}{\left|\widehat{e}_{2}\right|+\delta_{2}},
\end{aligned}
$$

where $\delta_{1}$ and $\delta_{2}$ are small positive constants.

The FTC algorithm is

$$
u=u_{0}+\Delta u_{f},
$$

where

$$
\begin{gathered}
u_{0}=\frac{1}{k_{2}}\left(\dot{\vec{e}}+\dot{\dot{r}}+\varepsilon \operatorname{sgn}(\widehat{s})+k \widehat{s}+k_{1} \widehat{x}_{2}+k_{3} F_{f}\left(\widehat{x}_{2}, t\right)\right), \\
\Delta u_{f}=-\frac{1}{k_{2}} \widehat{F}_{a} .
\end{gathered}
$$

The procedures of the system control algorithm are summarized as follows. 
Step 1. Construct the sliding state observer using (8).

Step 2. Calculate the estimated value of $e_{2}$ using (17).

Step 3. Calculate the reconfigured value of the generalized fault using (18).

Step 4. Calculate the FTC output using (19), in which $\Delta u_{f}$ is the rectified value that the controller uses to overcome the effect of the fault on the system.

\section{Experiments}

For a real-time control program it is more difficult to rectify online parameters and monitor the system in real time. Debugging is time consuming and expensive. Hardware-inloop (HIL) simulation and debugging are used to test and verify the effectiveness of the proposed control algorithm. In HIL simulation, the part that needs to be inspected represents the actual object and the other part is simulated digitally. Compared with pure digital simulation, this method is closer to real conditions. Simulink/xPC is used to carry out HIL simulation of our position servo system. xPCTarget is a product based on the framework provided by RTW (Real-Time Workshop) in MATLAB and this tool kit can provide a real-time simulation environment in which the host and target machines are independent from each other using standard PCs. In this environment, a model is built on the host machine using Simulink. The model is compiled as an executable file through RTW and a C compiler. The executable file is sent from the host to the target machine via serial port communication for operation. Thus, xPCTarget provides a precise approach for accomplishing simulation between two machines.

4.1. Configuration of the HIL Simulation System. In the environment MATLAB/xPCTarget, the real-time simulation system is constructed with the host machine and the target machine based on two microcomputers. The configuration is shown in Figure 2.

The host machine is a PC that controls the algorithm online and achieves real-time interaction during simulation. The PC is responsible for operating Matlab/Simulink/RTW to generate the code in real time, and performing interaction control and curve display functions during operation. The target machine is also a PC equipped with a GT-400SV controller with an I/O interface board. The core of the controller is an ADSP2181 digital signal processor and FPGA, which implements high-performance control and computation. The target machine is responsible for executing the code downloaded by the host machine and performing data exchange with the external object via the I/O channel. The external object comprises the DC servo motor, the drive circuit consisting of the isolated driver circuit and the power switch tube, and the incremental photoelectric encoder. This type of HIL simulation is also known as rapid control prototyping.
4.2. Experiments and Results. Under a Simulink environment, the control algorithm determined by (8) and (17), (18), and (19), and the position detection signal of a photoelectric encoder are delivered to a PC for real-time online control according to a given position signal. The control signal thus produced drove the DC servo motor using a drive circuit and simultaneously reconfigured the system fault.

During system operation, serial communication between the host machine and the target machine enables the host to receive a real-time feedback signal from the motor. The response curve is displayed in real time during system operation. The control parameters are also rectified in the Simulink environment according to the control state reflected by the curve until satisfactory system performance is achieved. Finally, an executable code is directly transferred from the host to the target machine, which is the core of the system control, and thus rapid prototyping of the control system is accomplished.

Since the system shows strong nonlinearity only under low-speed conditions, a low-amplitude and low-frequency sinusoidal signal are used as the given position signal, chosen as $r=0.1 \sin (2 \pi t)$ rad; the system noise is a uniformly distributed random number; the nominal resistance for the system is $R=7.77 \Omega$; and the control system parameters are $\delta_{1}=\delta_{2}=0.01, k=5, L_{1}=1, L_{2}=20$, and $c=30$.

The fault configuration ability and fault-tolerant performance of the system are tested by the following two types of faults.

Case 1. An incipient actuator fault is given as $F_{a}=\sin (4 t)$.

The experimental results obtained using xPC Target for Scope are shown in Figures 3, 4, and 5. From Figure 3 it is evident that in spite of the fault under the FTC mechanism the actual output signal of system $x_{1}$ rapidly tracks the given value immediately after $t>0.5 \mathrm{~s}$ and is no longer impacted by the fault.

Figure 4 shows the waveform for the actual speed and the observed speed value. It is evident that the observed speed value $\widehat{x}_{2}$ can rapidly track the actual speed value $x_{2}$, which is a good foundation for implementing fault reconfiguration and FTC.

Figure 5 shows the corresponding waveform for incipient fault reconfiguration. It is evident that, after $t>0.5 \mathrm{~s}$, good reconfiguration of the fault is obtained.

Case 2. An abrupt actuator fault is given by a step waveform.

When $t>1 \mathrm{~s}$, an abrupt fault occurs for which the amplitude is four times that in Case 1. The observer and controller parameters have the same values as in Case 1. Figure 6 shows the waveform of the fault reconfiguration. After $t>0.5 \mathrm{~s}$, good reconfiguration of the fault is obtained.

\section{Conclusion}

This paper presents a design for fault reconfiguration and FTC integrated in a built-in test system for electromechanical tracking of a servo platform. In comparison with fault 


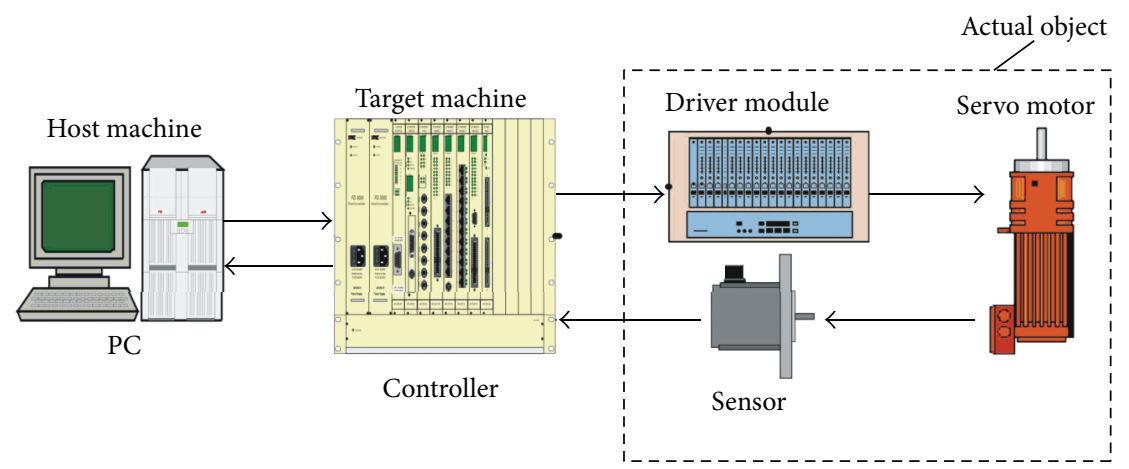

Figure 2: Configuration for the hardware-in-loop simulation.

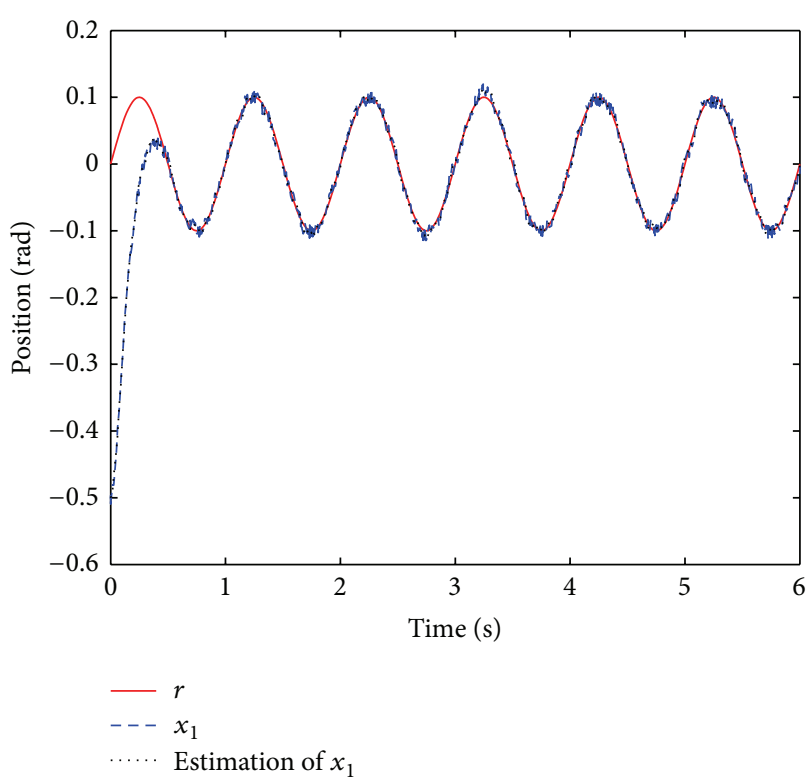

FIGURE 3: The given position and output position.

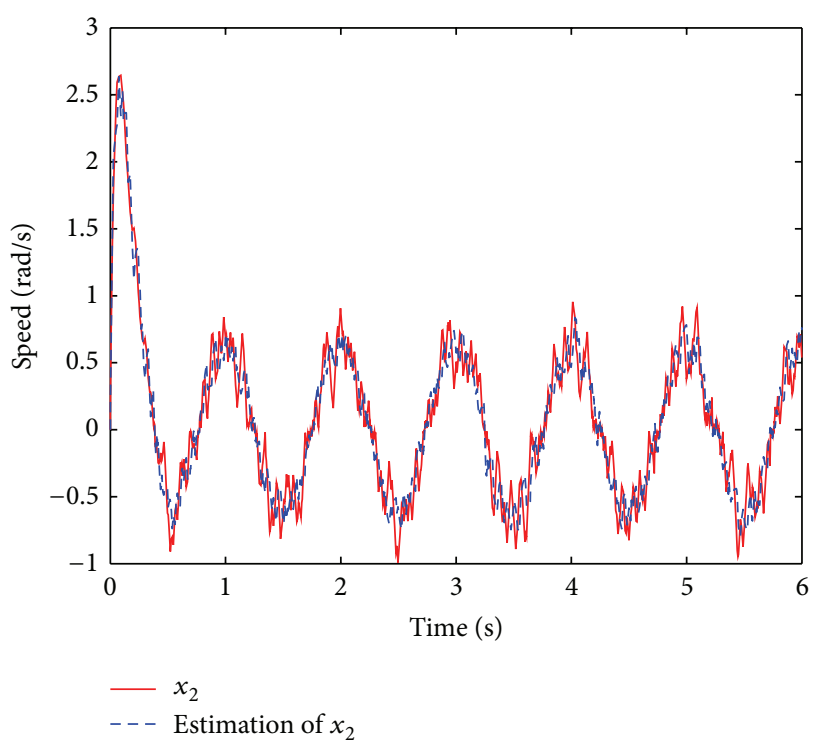

FIgURE 4: The speed and its estimation.

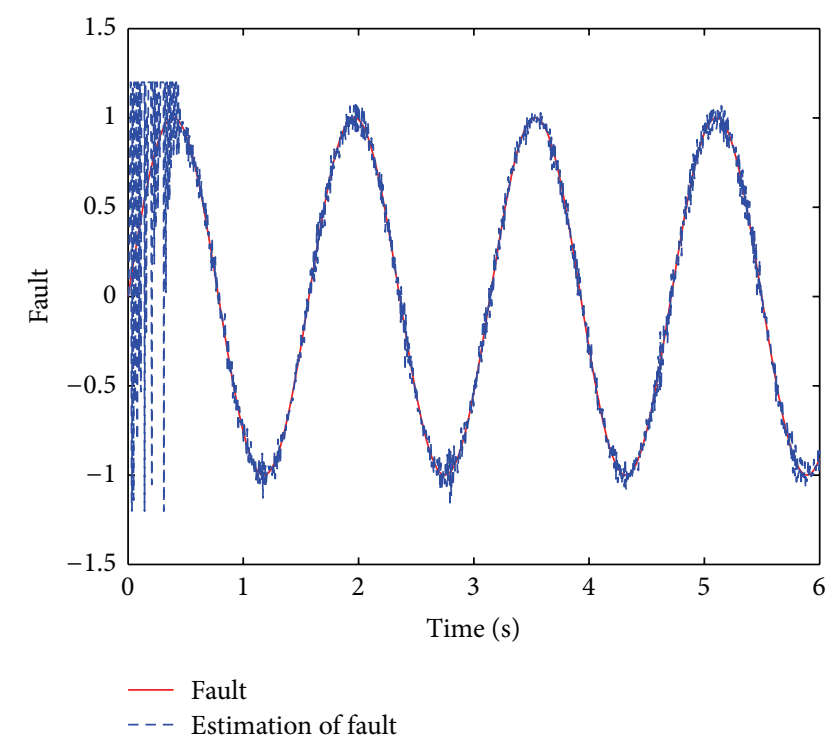

FIGURE 5: The incipient fault and its reconstruction.

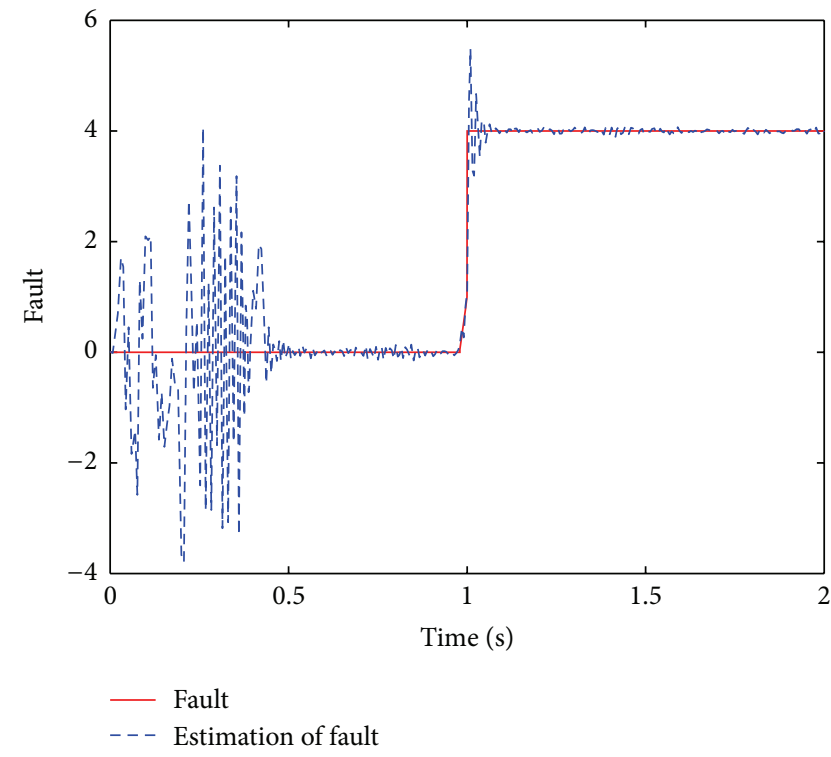

FIGURE 6: The abrupt fault and its reconstruction. 
detection by evaluation of residuals, the proposed fault reconfiguration approach better overcomes false or missing diagnoses for incipient faults. More importantly, the fault reconfiguration not only detects fault occurrences but also estimates the waveform and amplitude of the fault development, so that more precise fault diagnosis can be implemented. Furthermore, a simplified hardware configuration can be achieved by specific design of the state observer in which the observation of other state variables can be carried out as long as the value of one of them is known. Specific to this case, one angular displacement transducer is sufficient for data collection for the application example considered. The experimental results indicate that, in addition to efficient reconfiguration of actuator faults, the present algorithm allows the position output of the servo system to rapidly track the signal of a given position, even under fault conditions, which means that effective FTC is accomplished.

\section{Acknowledgments}

This work is supported by the Natural Science Foundation of China (nos. 61273157 and 61104024), Hunan Provincial Natural Science Foundation of China (no. 13JJ8020), and Hunan Province Education Department (no. 12A040).

\section{References}

[1] X. S. Wen, Y. C. Xu, X. S. Yi, and X. Chen, Theory and Application of intelligent Built in Test, National Defence Industry Press, Bejing, China, 2002.

[2] B. Armstrong-Hélouvry, P. Dupont, and C. C. de Wit, "A survey of models, analysis tools and compensation methods for the control of machines with friction," Automatica, vol. 30, no. 7, pp. 1083-1138, 1994.

[3] L. Xu and B. Yao, "Adaptive robust control of mechanical systems with non-linear dynamic friction compensation," International Journal of Control, vol. 81, no. 2, pp. 167-176, 2008.

[4] S. L. Chen, K. K. Tan, S. Huang, and C. S. Teo, "Modeling and compensation of ripples and friction in permanent-magnet linear motor using a hysteretic relay," IEEE/ASME Transactions on Mechatronics, vol. 15, no. 4, pp. 586-594, 2010.

[5] H. Chaoui and P. Sicard, "Adaptive fuzzy logic control of permanent magnet synchronous machines with nonlinear friction," IEEE Transactions on Industrial Electronics, vol. 59, no. 2, pp. 1123-1133, 2012.

[6] X. Li and W. Chen, "Application of active disturbance rejection controller for high precision servo system," in Proceedings of the International Conference on Mechatronic Science, Electric Engineering and Computer (MEC '11), pp. 2467-2470, Jilin, China, August 2011.

[7] S. Li and Z. Liu, "Adaptive speed control for permanent-magnet synchronous motor system with variations of load inertia," IEEE Transactions on Industrial Electronics, vol. 56, no. 8, pp. 30503059, 2009.

[8] V. Utkin, J. Guldner, and J. X. Shi, Sliding Mode Control in Electro-Mechanical Systems, Taylor \& Francis, London, UK, 1999.

[9] A. Šabanovic, "Variable structure systems with sliding modes in motion control-a survey," IEEE Transactions on Industrial Informatics, vol. 7, no. 2, pp. 212-223, 2011.
[10] S. L. Chen, K. K. Tan, and S. Huang, "Identification of coulomb friction-impeded systems with a triple-relay feedback apparatus," IEEE Transactions on Control Systems Technology, vol. 20, no. 3, pp. 726-737, 2012.

[11] S. W. Lee and J. H. Kim, "Robust adaptive stick-slip friction compensation," IEEE Transactions on Industrial Electronics, vol. 42, no. 5, pp. 474-479, 1995.

[12] Z. F. Gao, B. Jiang, P. Shi, M. S. Qian, and J. X. Lin, "Active fault tolerant control design for reusable launch vehicle using adaptive sliding mode technique," Journal of the Franklin Institute, vol. 349, no. 4, pp. 1543-1560, 2012.

[13] Y. Q. Wu, J. Z. Gu, and X. H. Yu, "Finite time sliding mode control for time delay systems," in Proceedings of the 4th World Congress on Intelligent Control and Automation, pp. 872-877, Shanghai, China, June 2002.

[14] X. Yu, B. Wang, and X. Li, "Computer-controlled variable structure systems: the state-of-the-art," IEEE Transactions on Industrial Informatics, vol. 8, no. 2, pp. 197-205, 2012.

[15] S. K. Spurgeon, "Sliding mode observers: a survey," International Journal of Systems Science, vol. 39, no. 8, pp. 751-764, 2008.

[16] G. Bartolini, A. Pisano, L. Fridman, and E. Usai, Modern Sliding Mode Control Theory, vol. 375 of Lecture Notes in Control and Information Sciences, Springer, Berlin, Germany, 2008. 


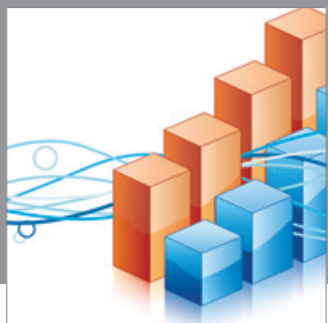

Advances in

Operations Research

mansans

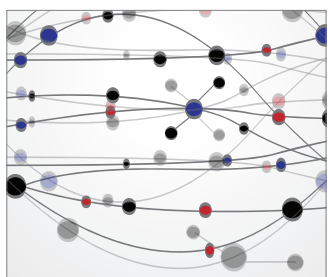

The Scientific World Journal
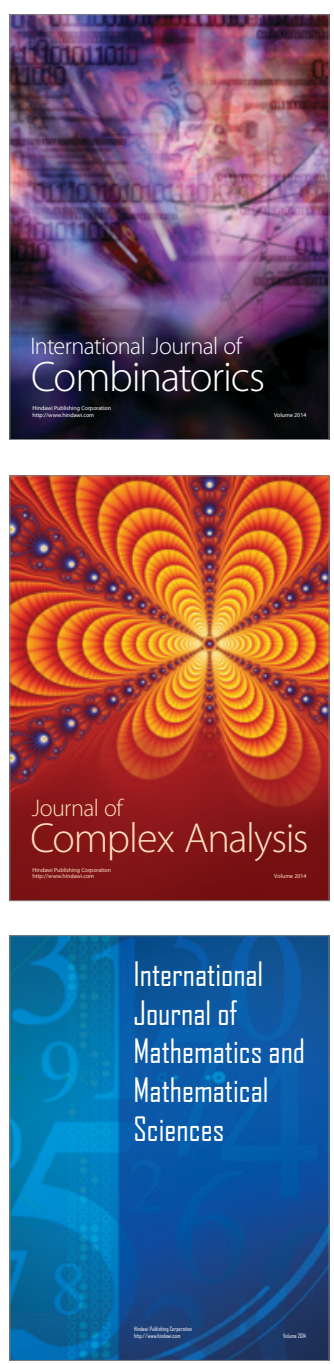
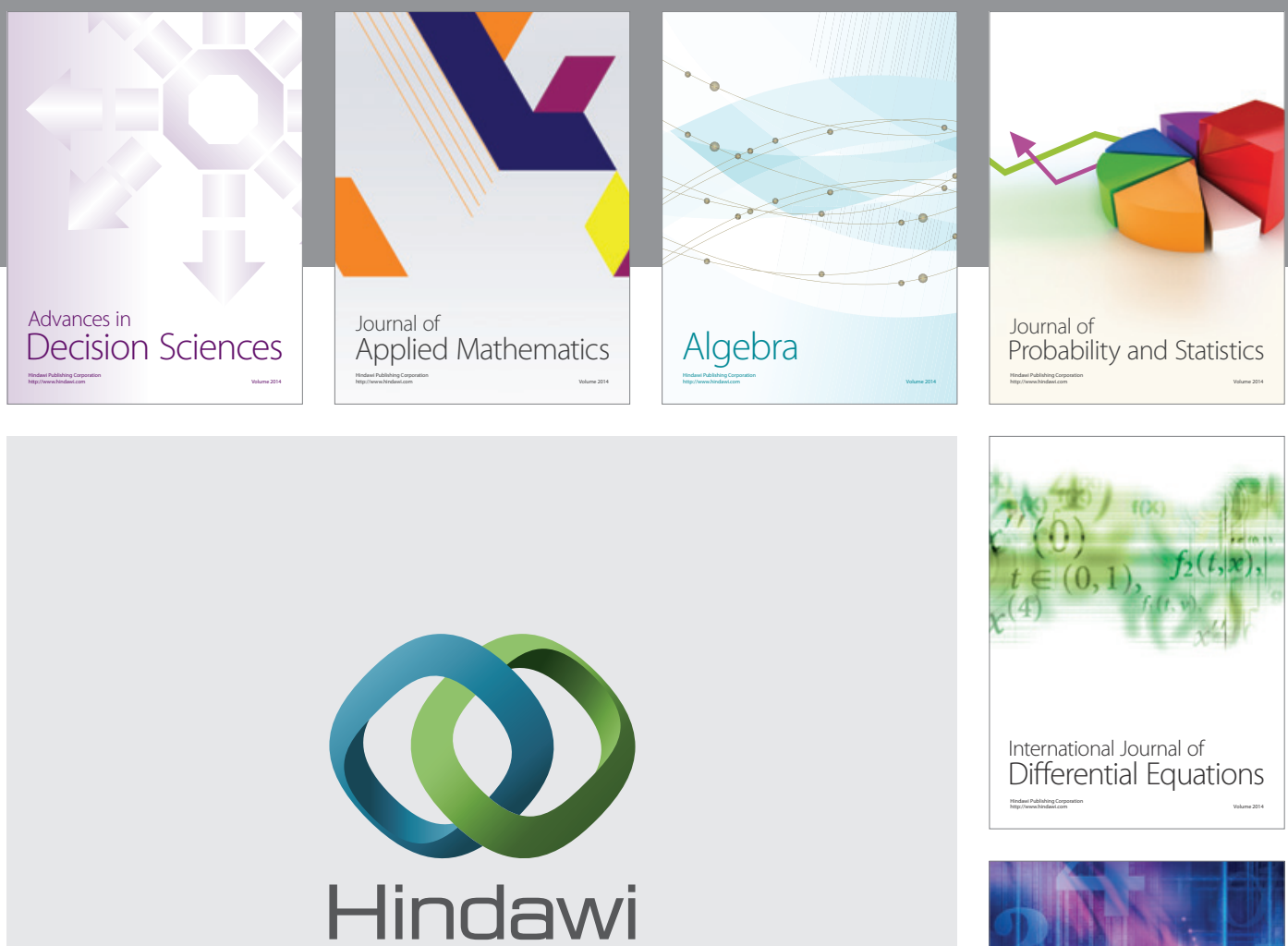

Submit your manuscripts at http://www.hindawi.com
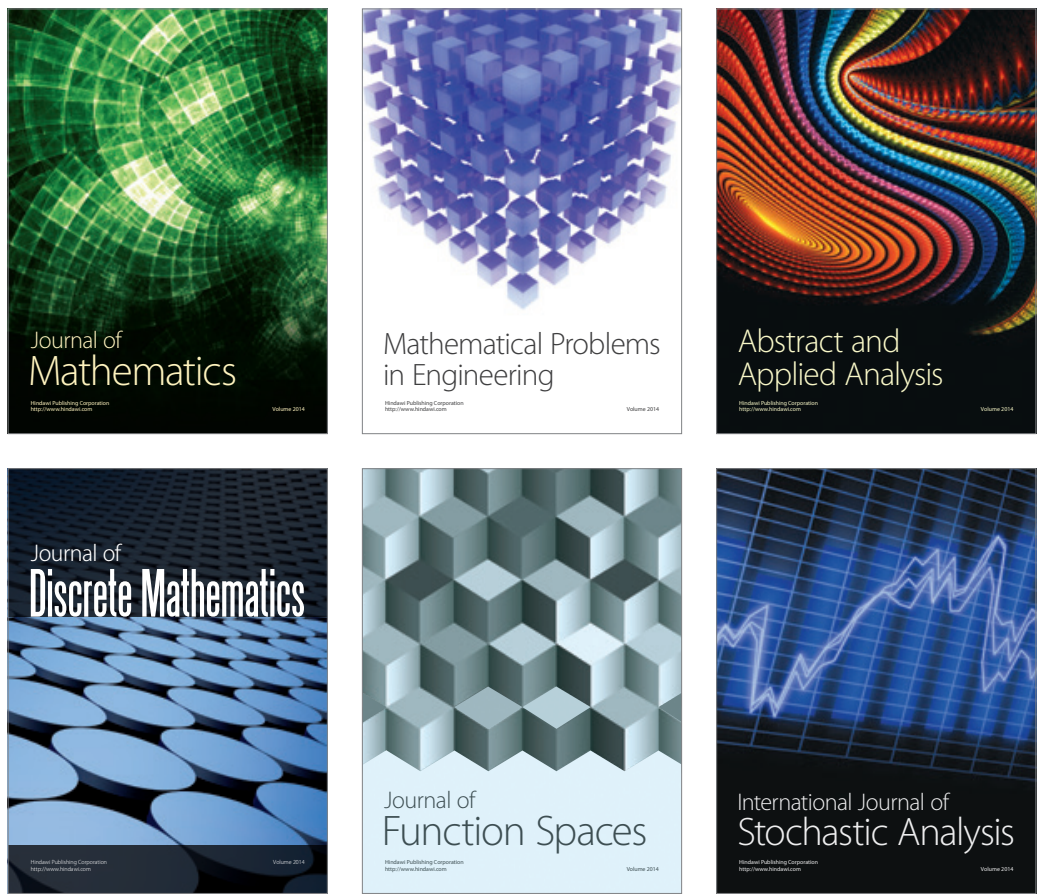

Journal of

Function Spaces

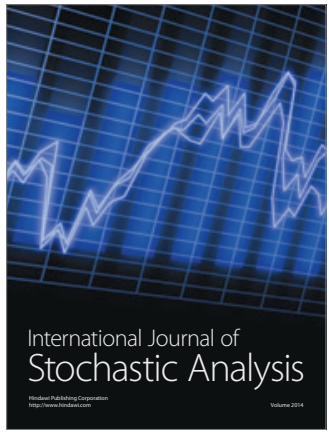

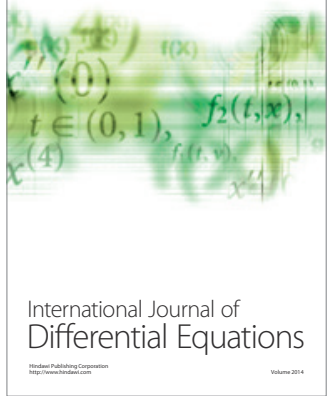
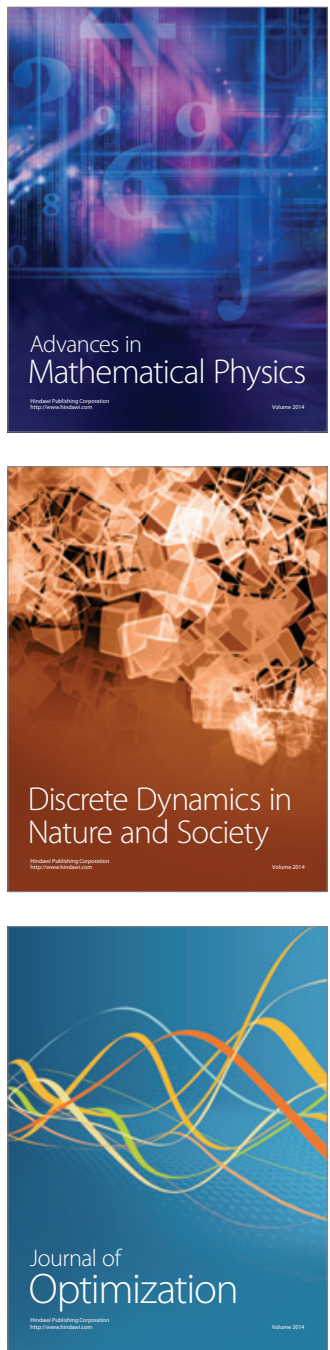